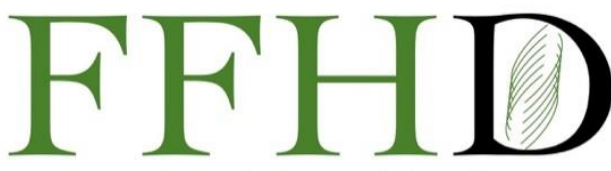

Functional Foods in Health and Disease

\title{
Marketing strategies for functional food products
}

\author{
Stella Chen ${ }^{1,2}$ and Danik Martirosyan ${ }^{2 *}$ \\ ${ }^{1}$ University of California, Berkeley, Berkeley, CA, USA; ${ }^{2}$ Functional Food Center, Dallas, TX, USA \\ *Corresponding author: Dr. Danik Martirosyan, PhD, ${ }^{2}$ Functional Food Center, Dallas, TX, USA \\ Submission Date: June 15 th, 2021 ; Acceptance Date: July 22 ${ }^{\text {nd }}$, 2021; Publication Date: August $5^{\text {th }} 2021$ \\ Please cite this as: Chen S., Martirosyan D. Marketing strategies for functional food products. Functional Foods in \\ Health and Disease 2021. 11(8): 345-356. DOI: https://www.doi.org/10.31989/ffhd.v11i8.817
}

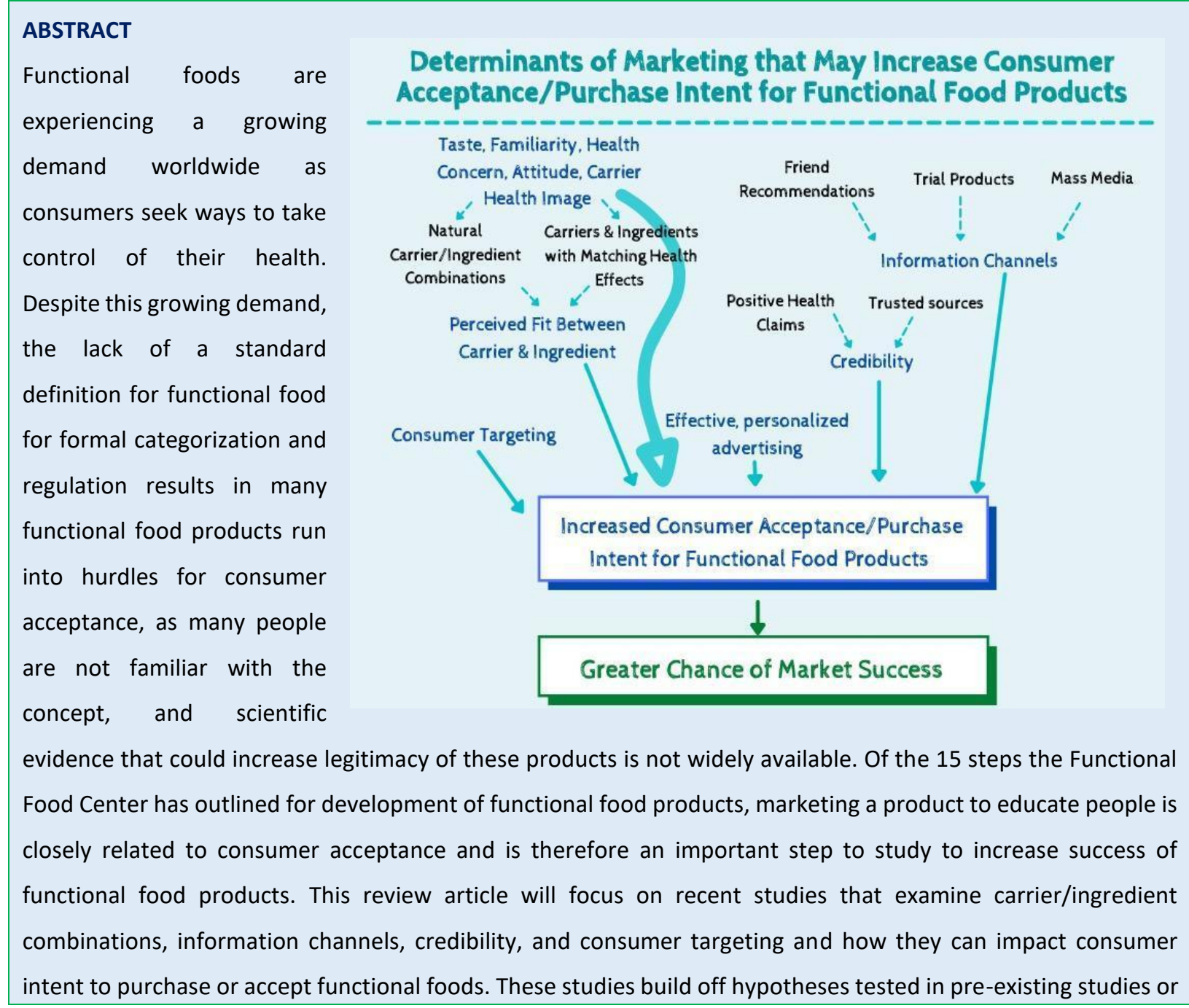


bring in novel determinants to consider. Finally, advice on marketing based on the results in the included papers and from an industry expert will be discussed.

Keywords: Functional Food Marketing, Carrier/Ingredient Combinations, Perceived Fit, Information Channels, Credibility, Consumer Targeting, Advertising, Consumer Acceptance, Purchase Intent

CFFC 2021. This is an Open Access article distributed under the terms of the Creative Commons Attribution 4.0 License (http://creativecommons.org/licenses/by/4.0)

\section{INTRODUCTION}

Functional food products are a combination of food and pharmaceuticals that bring together food science, nutrition, and medicine. There is not yet a standard definition for functional food, which has led to confusion among consumers, developers, and the government. Having a standard definition is important for developing and promoting functional foods and for consumer acceptance of functional food products, because without a definition, they cannot be categorized or regulated formally. In order to expand acceptance, prevent misinformation, and advance functional food as a separate category, the Functional Food Center (FFC) currently defines functional foods as "natural or processed foods that contain biologically-active compounds; which, in defined, effective, non-toxic amounts, provide a clinically proven and documented health benefit utilizing specific biomarkers, to promote optimal health and reduce the risk of chronic/viral diseases and manage their symptoms" [1]. Furthermore, Dr. Martirosyan of the FFC has outlined 15 steps for the development of functional food products and for bringing them to the market [1]. This is a long process, but if widely recognized, can serve as a guide to functional food scientists, researchers, and companies around the world. These steps are outlined below:

1. Establish the goal of the functional food product.

2. Find the relevant bioactive compound.
3. Establish a dosage for the bioactive compound.

4. Establish the pathway and molecular mechanisms of the bioactive compound.

5. Find relevant biomarkers related to the mechanism and pathway.

6. Create a food vehicle containing the bioactive compound.

7. Provide preclinical studies on dosage, efficacy and safety.

8. Provide clinical trials for dosage, efficacy, and safety.

9. Create a special functional food label that includes consumption instructions, dosage recommendations, and shelf life.

10. Market the functional food product to educate people.

11. Provide pilot and/or epidemiological studies of the functional food product. This is crucial for building trust with the public.

12. Send information to a government agency or trusted third-party organization for approval.

13. Release the functional food product to the market.

14. Provide after-market research. This will monitor for any gaps between controlled study results and real-world results.

15. Final establishment of the functional food product.

Below is an infographic summarizing the steps for functional food development. 


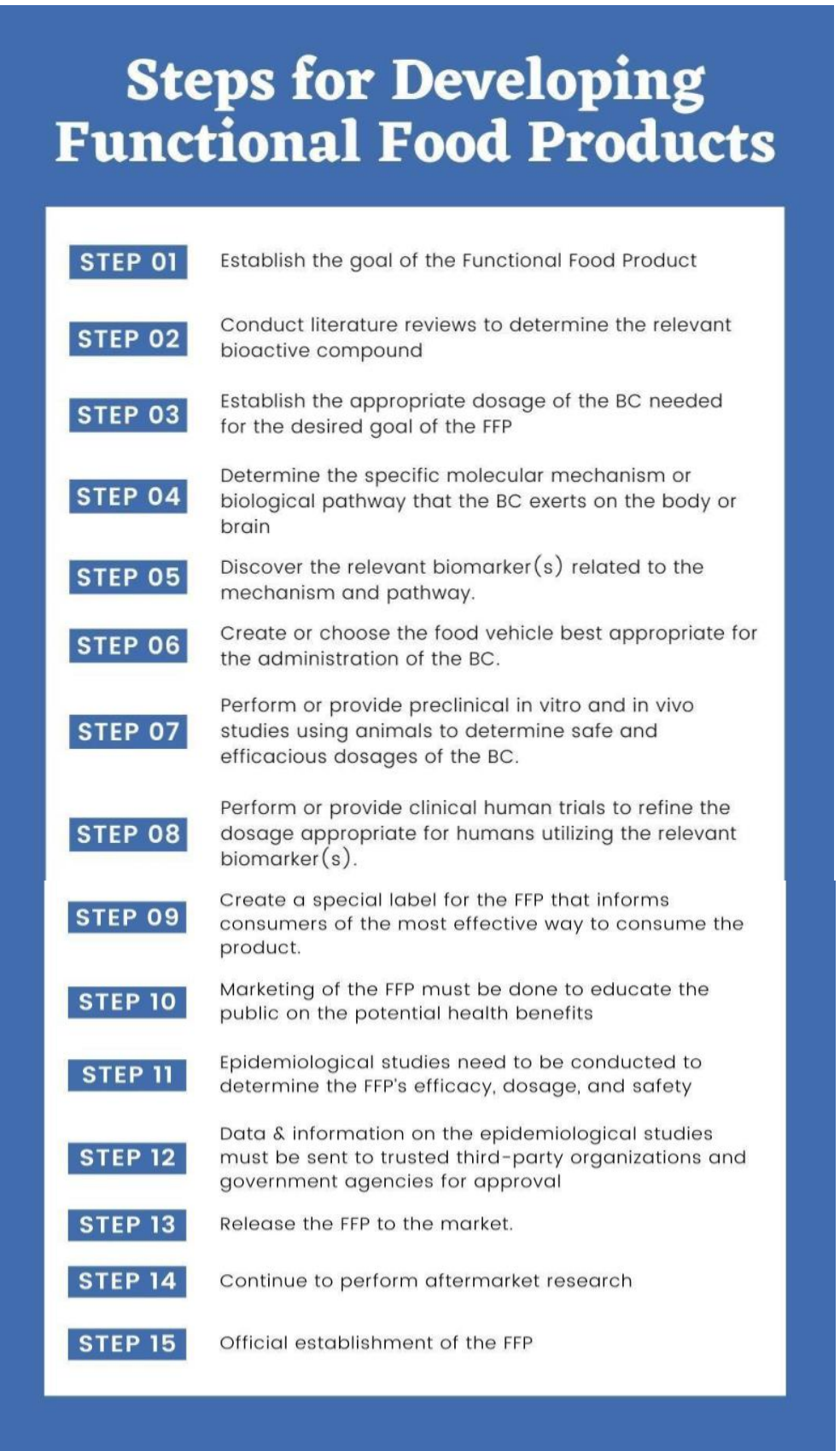

Figure 1 [1]. Steps for Developing Functional Food Products: $B C=$ bioactive compound; FFP= functional food product

This review article will focus on Step 10, marketing of functional foods to educate people. The functional food market was valued at $\$ 300$ billion in 2017 and is predicted to reach $\$ 440$ billion in 2022 , although these values are in dispute; the lack of a definition for functional food leads to different classifications on what is a functional food product and what is not, leading to differing market value estimates [2]. In
Japan, where there is a consensus on functional foods as a separate and recognized category, the functional food market amounted to $\$ 8$ billion in 2018 , the latest year that the United States Department of Agriculture has data for [3]. Either way, there is consensus that the functional food market is continuing to grow and expand, and it will be highly beneficial for companies to advertise their functional food products effectively 
to take full advantage of this growing market. Research into consumer attitudes and other factors affecting purchase intent towards functional foods can help with formulating marketing strategies.

There are already several studies that have been done in past years about what influences consumers to purchase functional foods. Although health is one of the criteria that consumers may consider when purchasing a functional food, it is not the only criterion. Merely counting on consumers' willingness to trade off taste for health is risky [4-5]. In fact, taste has been marked as an especially important factor when determining consumer purchase intent [6-7]. Other studies have been done on how carrier/ingredient combinations and familiarity with the product can affect acceptance of functional foods [8-9].

This review will examine recent studies that build off hypotheses by the aforementioned studies, as well as test other determinants of functional food acceptance that have not been examined yet. Advice on marketing functional foods will be covered based on study results.

\section{REVIEW}

Carrier/Ingredient Combinations: There are a myriad of factors that can influence acceptance of functional foods by consumers. Health concerns and attitudes towards functional foods are some factors that can influence consumer's purchase intent and in 2011, Krutulyte et al. examined another determinant in addition to the ones above: "perceived fit of carrier/ingredient combination" [10]. They hypothesized that the more a carrier food fits with the bioactive compound, or "ingredient" added to it, the stronger the consumer's intention to buy the functional food will be. The healthy image of the carrier expected taste of the ingredient, consumer's familiarity with the carrier and ingredient, and the naturalness of the ingredient in the carrier, feed into the factor of "perceived fit of carrier/ingredient combination". Krutulyte et al. continues past studies that have investigated the impact of carrier/ingredient combinations on consumer acceptance of functional food $[8,11]$.

959 surveys were collected from the Danish population. General attitudes towards functional foods and health concerns were assessed by having participants answer statements about opinions on functional foods and self-assess their risk of getting sick. Intention to buy functional foods due to perceived fit of carrier foods and ingredients were assessed as well, with respondents answering "Yes" or "No" to whether a certain combination made sense and whether they would purchase it or not. The carrier foods in the survey included yoghurt, muesli bars, fish balls, tuna salad, baby food, rye bread, and bacon liver pâté. The ingredients were omega-3, fish oil, vitamins, minerals, plant sterols, and fiber. The carriers and ingredients were arranged into 42 combinations. Finally, 20 experts from Danish nutritional institutions were also asked to rate carrier/ingredient combinations based on market familiarity, taste of the combination, the overall quality of the combination, and the perceived healthiness of the carriers, in order to analyze consumer responses [10].

Based on consumer responses, carrier/ingredient combinations do influence purchase intent. The most attractive carriers were muesli bar, yoghurt, and rye bread while the most attractive ingredients were plant sterols, vitamins, and minerals. Ingredients with no taste connotation, such as vitamins and minerals, seem to have a better perceived fit with carriers than ingredients that may have taste impact such as fish oils and omega-3. 
Furthermore, the most popular combination was tuna salad with fish oil with a logistic regression of $\mathbf{1 3 . 9 7 5}$ for intention to buy and $\mathbf{1 2 . 0 6 0}$ for perceived fit $(p<0.01)$. Compared to a combination like yoghurt and plant sterols ( 0.467 for intention to buy, 0.455 for perceived fit; $p<0.01)$, the more popular combinations suggest that consumers prefer pairings that are "natural" [10]. For instance, fish oil is present in tuna salad already, so adding more fish oil to tuna salad is a natural addition, which consumers seem to accept more readily.

When evaluating different factors' impact on purchase intent, health concern had the weakest impact with a logistic regression of $1.031(p<0.05)$. Attitude towards functional foods was higher at 1.118 $(p<0.01)$. Perceived fit of carrier/ingredients had the biggest impact; if the carrier and ingredient were not perceived as a fit by consumers, acceptance of the product dropped to 0.038 from a fixed parameter of $1.0(p<0.01)[10]$. These results show that it is important for functional food developers to fit natural combinations of carriers and ingredients together as this factor may play a larger role in purchase intent than health concern and attitude.

The more familiar consumers are with the combinations, the more likely they are to buy it. For example, rye bread and omega-3 are not necessarily a natural combination, but since rye bread is a familiar food for Danish consumers, the logistic regression for this combination was higher than expected at 0.458 for intention to buy and 0.430 for perceived fit $(p<0.01)$ [10]. Finally, with the help of the expert rankings, consumers tend to perceive a better fit if the carrier had been categorized as less healthy by experts. More investigation is needed into this conclusion though as past studies have disagreed on how perceived health of the carrier impacts purchase intent. Overall, the Krutulyte et al. study suggests that natural carrier/ingredient combinations using familiar ingredients can facilitate consumer acceptance of a functional food product in the market. Functional food producers should conduct research about what foods their target audiences are familiar with as this study was specific to just the Danish population.

In 2019, Temesi et al. built on Krutulyte et al.'s research on perceived fit of carrier/ingredients. They introduced a new determinant: "perceived correspondence of health effects" where the carrier and ingredient does not necessarily need to be a natural combination like Krutulyte et al. suggested. Instead, it is more important that their health effects be similar [12]. For this study, 1,016 Hungarian respondents answered a 10-minute questionnaire. The sample was not representative with most respondents being women between the ages of 1829 who were highly educated (secondary or tertiary education). 5 carriers and 11 ingredients were arranged into 28 unnatural combinations and rated on perceived healthiness of the carrier, taste, and familiarity with the combination. Respondents were also to pick statements about health effects that they associated with each carrier and ingredient to measure "perceived correspondence of health effects" and evaluate how much the combinations fit with each other in terms of health effects.

Taste was determined to be the most important factor for determining "perceived correspondence of health effects" in all examined combinations. It plays an especially important role with yoghurt where the chance that a consumer perceives a fit between yoghurt and the added ingredient is 4.3 times higher if the expected taste is good [12]. Awareness is also important for perceived fit and acceptance. If the respondent already knew of the carrier/ingredient combination, they are more likely to regard it 
favorably. Thus, marketing may be especially important to expose consumers to carrier/ingredient combinations in order to increase consumer acceptance of a functional food product. In addition, unlike the Krutulyte et al. study, if the carrier was perceived to be healthy, consumer purchase intent increased $[10,12]$.

Correspondence of health effects could not be measured for dark chocolate and ginseng. Ginseng is not an ingredient that Hungarians are familiar with while for dark chocolate, the taste may mask the importance of nutritional content to consumers. Therefore, dark chocolate, other foods that fall in the "fun foods" category, and unfamiliar foods are not recommended for use as a carrier [12].

Temesi et al. also examined sociodemographic characteristics and its impact on functional food acceptance. On average, good taste resulted in a 3.6 times bigger chance of perceived fit especially among those with primary education, those with below average income, those older than 30 , and people from the Hungarian capital. Awareness of the carrier/ingredient combination was especially important in women, young people, and those from the capital. Lastly, the health image of the carrier and perceived correspondence of health effects increased the perceived fit of carrier/ingredient combination by $30 \%$ and were especially important to women, those with secondary education, and those with an average income [12].

Although different groups had different priorities when it came to accepting functional food, Temesi et al. affirms the importance of taste for consumer acceptance. Awareness of the product and perceived correspondence of health effects were positive influencing factors as well. Thus, developers and marketers should focus on these determinants to ensure success of their product. It should be noted that unlike Krutulyte et al., the ingredients do not need to be present in the carrier as natural combinations. Instead, more emphasis should be placed on matching health effects between the carrier and the ingredient.

Carriers and Information Channels: A 2020 study done in China by Huang et al. brings in additional factors to study for consumer acceptance of functional foods. They explore the effects of carriers on purchase intent and introduce a new determinant: information channels. China is a promising market for functional foods as it has had a long history of using food to prevent and treat chronic diseases. Thus, there is already public interest and some national/governmental programs in place for functional foods [13]. Being able to predict what products consumers may accept will be very advantageous in the Chinese functional foods market.

In this study, 1,144 questionnaires were administered in 16 regions in China. Most respondents were younger, had higher education, and were from urban areas. 3 carrier foods were used: yogurt, a non-alcoholic beverage, and biscuits. The study's authors evaluated perceived attractiveness and purchase intention towards these 3 carriers. Respondents were also asked how much trust they had in different information channels about functional foods.

Yogurt scored the highest on perceived attractiveness and purchase intention with a mean value of 3.30 and 3.28 out of 5 , respectively $(p<0.05)$. Biscuits had the lowest values at 2.85 for perceived attractiveness and 2.87 for purchase intention $(p<0.05)$. In this case, the healthier a carrier is, the higher the purchase intent, which disagrees with the findings of the Krutulyte et al. study but agrees with Temesi et al. [10, 12-13]. Improving immunity and 
improving gastrointestinal function were perceived by consumers as the most attractive functions $(p<0.05)$ [13]. At least for the Chinese population in general, functional food products that use yogurt as carriers and that focus on improving immunity or gastrointestinal function may be favorable and guarantee higher chances of product survival in the market.

There are differences among groups, however. Respondents above 55 and middle to high-income groups were attracted to reducing cardiovascular disease and osteoporosis risk. Females were attracted to improving facial skin health and reducing body fat. Married respondents had higher attraction towards delaying senescence and reducing cardiovascular disease risk. Respondents with low education perceived the least attraction and purchase intent for all benefits and carriers. Urban respondents showed generally higher attraction and purchase intent for functional foods although it was only significantly higher, compared to rural respondents, for the function of delaying senescence. These results can inform developers on what benefits to focus on, depending on what audience they are targeting. However, the scientists concluded that functional foods for improving overall health are more suitable for the Chinese market due to the mass-market appeal. Marketing products with specific benefits carry more risk as they only appeal to small market niches [13].

On average, consumers trusted friend recommendations the most when it came to accepting functional foods, with the average mean score being a 3.66 out of 5 . Trial products where consumers can test out a product before buying were the second highest at 3.08, and TV was the third highest at 3.06. Although mass media (TV) is not the most influential, the study authors continued to emphasize the importance of improving advertising to increase consumer acceptance and trust for a product. Second, trial products, although they are expensive to market, create a pre-purchase experience that can convince consumers to buy unfamiliar products by reducing uncertainty and increasing demand. Finally, more attention should be paid to interpersonal networks and further research can be done on the role of opinion leaders in networks, for instance, to determine how marketing and product development can cater to this factor of friend recommendations [13].

Credibility: Credibility is another important factor for functional food acceptance and can affect consumer willingness to purchase and pay for a product. Plasek et al. conducted a literature review in 2019 examining this relationship. They found that health claims were important for influencing consumer purchase, especially among highly involved consumers who pay attention to such claims. Claims that suggested positive health benefits (i.e., reduced cholesterol levels) positively influenced willingness to pay while claims about potential risks (i.e., reduced risk for cardiovascular disease) garnered less attention and were regarded as less credible [14]. In general though, health claims, especially positive health claims, increase willingness to pay by adding to a product's credibility. This is particularly important for those who are not familiar with the ingredients.

Providing information in health claims usually makes a product more credible but sometimes, this can backfire. In a case where consumers have less trust in a technology or development, providing information may lower willingness to pay [14]. For example, if information is provided states that a product had been manufactured with the help of nanotechnology, and consumer trust in 
nanotechnology is low, willingness to pay will decrease. Therefore, companies must be aware of consumer attitudes towards the technology used in development of their product.

The source of information is essential for determining credibility. Consumers consider dietitians as fairly credible while trusting food companies less. Social media was considered the least credible while promotions in health magazines were ranked in the middle [14]. The person used in the advertisement also has an effect. Celebrities were regarded as the least credible while established health organizations (i.e., Dutch gastro-intestinal association) were the most reliable. Further research also found that featuring a lean person in functional food advertisements made the product more credible for consumers. A lean build is considered healthy, and consumers associate that with consumption of the advertised product [14]. Companies should pay attention to what avenues and which communicators they are using to advertise a product. Utilizing medical professionals, credible organizations, and even lean, healthy people can increase credibility of a functional food product to consumers.

Plasek et al. point out the need for further studies on functional food credibility, referencing studies that have disagreed on whether credibility affects willingness to purchase and pay for a product. Further research is also needed about the factors that influence the credibility of functional foods. Despite limited information and conflicting studies, Plasek et al. maintain the importance of credibility for consumer willingness to purchase and pay for functional food products.

Consumer Targeting: In 2018, Moons et al. conducted a case study on targeting certain groups for Spirulina-enhanced food; this can inform companies of the efficacy of targeting specific consumer groups. Spirulina is a microalgae that is used in functional foods and is a good, environmentally friendly alternative to meat. The researchers surveyed sporting, vegetarians, and 'foodie' (those who follow the latest food trends and visit trendy restaurants and shops) consumers and compared them with a contrast group of life-enjoyers (those who prioritize taste and pleasurable aspects of food and who tend to adhere to traditional foods). These 4 groups were surveyed on health consciousness, neophobia (fear of new foods), willingness to compromise on taste, and environmental concern. How these factors feed into Spirulina food adoption intention was examined, and the consumers were asked at the end whether they would purchase Spirulina-enhanced foods [15].

1,325 Belgian respondents completed the survey. The results are as follows: life enjoyers had the significantly lowest adoption intention $(p<0.05)$ while foodies' adoption intention was significantly the highest $(p<0.05)$. Sporting and vegetarian adoption intentions did not differ significantly $(p=0.88)$. Health consciousness was a significant and positive factor for adoption in all groups, with lifeenjoyers having the smallest positive effect $(p<0.01)$. Food involvement only positively influenced sporting and life-enjoyers and only the life-enjoyers effect was significant $(p<0.05)$. Food neophobia was significant in foodies and life-enjoyers only $(p<0.05)$ for decreasing purchase intent. Environmental concern was not significant in any group [15].

The researchers commented on the results above. Sporting individuals were less interested in food than expected; while they are health-conscious, they may not necessarily be interested in using food to improve health. Sporting individuals were also not affected much by neophobia in the case of Spirulina, 
because they may be used to taking food supplements that are protein rich. Spirulina is protein-rich so it is similar to protein-enhanced supplements that sporting individuals may already take. When marketing to sporting individuals, the researchers recommend focusing on how food can achieve a better body shape and improve physical performance. Advertising should be done at sports events, sports clubs, and sports stores [15].

Vegetarian consumers were surprisingly less concerned with environmental affects and were actually motivated more by health-consciousness. They were not affected much by neophobia either because of their general interest in replacing meat and openness to new options. When marketing to vegetarians, focus should be on meat and dairy-free protein sources with specific, beneficial health effects. One can advertise at vegetarian restaurants and at specialized events directed towards vegetarians to reach them [15]. Overall, both vegetarians and sporting individuals are more health conscious and more willing to compromise on taste, which marketers should take into account.

Foodies are more health-conscious than expected, although they prioritize taste much more than sporting and vegetarian individuals. Foodies are also more neophobic than previously thought. When marketing to foodies, it can be helpful to inform them that functional food is a means to stay healthy and have a happy life, as foodies prioritize these. Avoid "novelty" and "compromised taste" approaches as the surveys showed that this decreases purchase intent in foodies. For foodies, marketing through websites, magazines, TV cooking programs, and food fairs/exhibitions is advised [15].

Environmental arguments should be avoided for these groups as it had no significant impact. Gender and age had no effect on adoption intention either among these groups except in the vegetarian group where women were more likely to adopt Spirulina than men [15]. This case study shows that personalizing marketing techniques to groups that are likely to adopt a functional food product can be beneficial to a product's survival in the market, as they can contribute to early adoption of the product. It is important that further research is done into motivational drivers and barriers to acceptance of functional foods in these early-adopter groups to ensure product success.

Advice from an Industry Expert: Lastly, in 2017, Jeff Hilton, who has been in the functional foods industry for 25 years, provides some advice on marketing functional foods that can add on to the advice provided in the studies mentioned above. He points out the potential for functional foods. Functional foods bring together food and dietary supplements in a 2-for-1 mentality, alleviate pill fatigue especially among older baby-boomer consumers, correspond with a rise in health awareness, is convenient, and simplifies life and nutritional intake for consumers [16].

According to Hilton, despite this potential, many products fail because there is not enough consumer promotion, education, and market differentiation. In addition, many final functional food products lack effective dosages and legitimate research into product efficacy, which leads to lack of perceived and actual efficacy in these products. Before marketing, the quality of the product must be up-to-par.

As for marketing, companies need to decide whether to brand their product or not. Branding can help differentiate the product but should only be done so if appropriate. Hilton recommends branding if the manufacturing company possesses valuable trade secrets/patents, has scientific support for their ingredient, is planning on doing business in substantial, active categories, and/or has potential 
co-branding partners to share the risk and invest in the brand [16]. Furthermore, science is important. Product packaging can be revised to be more sciencedriven, such as including scientific information with the product. Information can be administered using stories and metaphors to keep consumer attention [16]. For example, an unhealthy endothelial GI lining can be compared to flypaper that cholesterol and fats stick to, which leads to plaque buildup and blocks arteries. The analogy with flypaper facilitates consumer understanding. Moreover, visuals, audio, and video media can all be used to impart information and reach different parts of a consumer's brain for more effective advertising. Companies can also consider using a healthcare practitioner or other expert to endorse their product. Finally, one can expose consumers to information at the right time. Functional foods can be advertised at doctors' offices (i.e., infographic or video in the waiting room), and information can be available to consumers $24 / 7$ so anyone can access the information on their own time [16].

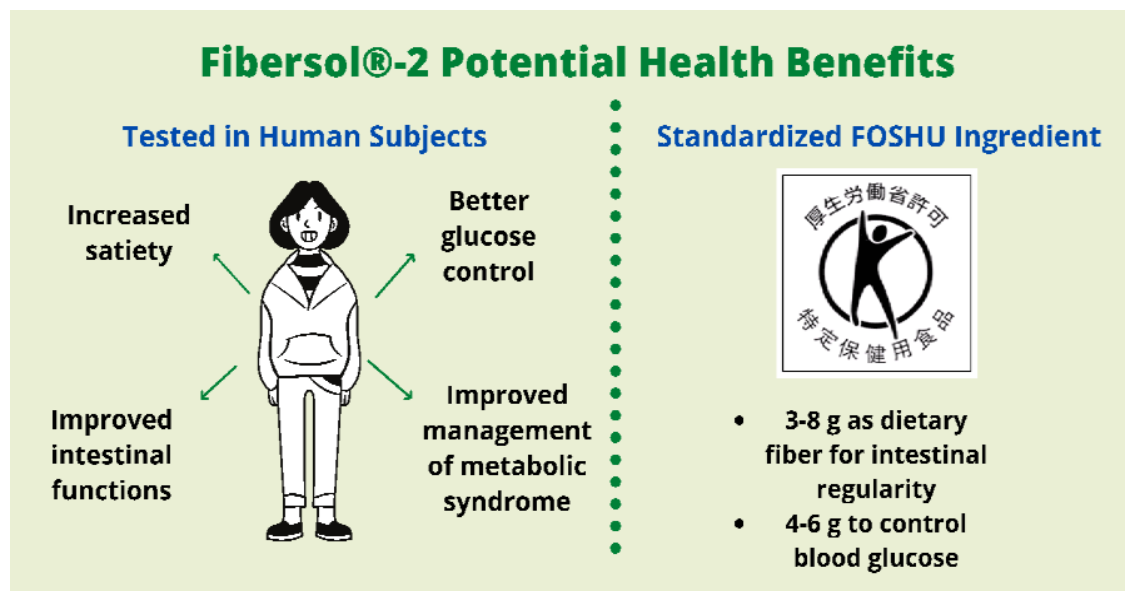

Figure 2 [17]: An example of an infographic that uses some of Hilton's different advertising strategies. This is an infographic for the functional food product, Fibersol ${ }^{\circledR}-2$. It uses images, words, and color-coding to reach different parts of a consumer's brain and the written information is brief and easy to understand.

Different demographics have different preferences when it comes to functional food delivery. Senior citizens like powders to mix into or sprinkle onto foods as well as liquids and sublinguals (food that is applied under the tongue). Gen X prefers gels and strong flavors while millennials like gummies, shots, and chewables [16]. Millennials will soon become the largest global demographic segment and companies should take them into consideration during marketing. Millennials tend to prefer authenticity, transparency, are focused on enhancing lifestyle, and are best reached through social media and storytelling marketing strategies [16]. In short, marketing should be relevant to target populations; one-size-fits-all approaches are not as effective.

Below is a summary of strategies companies can use to improve marketing of their functional food product and raise the chances of product success.

1. Use natural carrier/ingredient combinations that consumers are familiar with [10].

2. Do not use "fun foods" such as dark chocolate as a carrier in functional foods because taste undermines the importance of health and does not increase acceptance of the product as a functional food. Avoid unfamiliar carriers as well [12]. 
3. Taste is most important for consumer acceptance of functional food [12].

4. The carrier and ingredient should have matching health effects [12].

5. At least for the Chinese market, marketing foods with general health benefits are more suitable due to mass-market appeal [13].

6. Effective advertising in mass media, increasing availability of trial products, and studying the effects of friend recommendations are important for improving consumer acceptance of functional foods [13].

7. Health claims may focus more on positive benefits rather than reduced risk of disease [14].

8. Using dietitians, other healthcare professionals, and people with lean builds can increase credibility of a functional food product and increase acceptance [14].

9. Targeting specific consumer groups can lead to early adoption of a functional food product and increase the chances of the functional food product surviving in the market [15].

10. Brand your product if appropriate. This can help with product differentiation [16].

11. Use stories and different forms of media to advertise and impart information on functional foods [16].

12. Personalize your product development (for example, if your functional food is directed at seniors, use powders, liquids, or sublinguals as seniors have been shown to prefer these) [16].

13. Time and place your advertising carefully [16].
More investigation is needed on many of these points. For example, Temesi et al. disagrees with Krutulyte et al. about natural carrier/ingredient combinations, asserting that as long as health effects are similar, it does not matter how natural the combination is. Nevertheless, each of the points above have been proven by study authors to be effective on their subjects and is worth trying in larger and more diverse populations to assess efficacy.

\section{CONCLUSION}

This review has brought together several recent studies that examine different aspects of marketing that influences consumer purchase intent for functional foods. Studies that added on to previous ones about the carrier/ingredient combination's influence on consumer purchase intent were analyzed. Information channels, credibility, and consumer targeting, which are lesser examined determinants that warrant further study, were investigated as well. While in general, there is a need for further research (as there are conflicting results among papers), these studies offer concrete strategies for companies to try, backed up by scientific evidence. It is estimated that around $90 \%$ of new functional food products fail, so investigation into marketing strategies that can reduce the failure rate is much needed and can benefit a world where lifestyle-related conditions are on the rise $[14,18]$. Definitive conclusions on marketing strategies can improve the development and marketing process for functional food development companies and improve quality of life for many consumers.

List of Abbreviations: FFC: Functional Food Center, BC: bioactive compound, FFP: functional food product 
Competing Interests: The authors have no financial interests or conflicts of interests.

\section{Authors' Contributions: Dr. Martirosyan devised the} topic of this review article and provided detailed guidance and review of the research, writing, and editing process. Stella Chen narrowed down the subject, collected the data, and wrote the review article.

Acknowledgements: No external funding was needed or given for this review article.

\section{REFERENCES}

1. Martirosyan D, Kanya H, Nadalet C: Can functional foods reduce the risk of disease? Advancement of functional food definition and steps to create functional food products. Functional Food in Health and Disease 2021, 11(5): 213-221. DOI: https://www.doi.org/10.31989/ffhd.v11i5.788

2. U.S. Functional Foods Market- Statistics \& Facts [https://www.statista.com/topics/1321/functionalfoods-market/] Retrieved June 2, 2021.

3. Japan Health Foods Market Overview (USDA) [https://apps.fas.usda.gov/newgainapi/api/Report/Do wnloadReportByFileName?fileName=Japan $\% 20$ Health \%20Foods\%20Market\%20Overview Tokyo\%20ATO Ja pan_03-29-2020] Retrieved March 31, 2021.

4. Cox DN, Koster A, Russell CG: Predicting intentions to consume functional foods and supplements to offset memory loss using an adaptation of protection motivation theory. Appetite 2004, 43(1): 55-64. DOI: https://doi.org/10.1016/i.appet.2004.02.003

5. Verbeke W: Functional Foods: Consumer willingness to compromise on taste for health? Food Quality and Preference 2006, 17(1-2): 126-131. DOI: https://doi.org/10.1016/j.foodqual.2005.03.003

6. Nielsen NA, Bech-Larsen T, Grunert KG: Consumer purchase motives and product perceptions: a laddering study on vegetable oil in three countries. Food Quality and Preference 1998, 9(6): 455-466. DOI: https://doi.org/10.1016/S0950-3293(98)00022-6

7. Lyly M, Roininen K, Honkapää K, Poutanen K, Lähteenmäki L: Factors influencing consumers' willingness to use beverages and ready-to-eat frozen soups containing oat $\beta$-glucan in Finland, France and Sweden. Food Quality and Preference 2007, 18(2): 242255. DOI:
8. Bech-Larsen T, Grunert K: The perceived healthiness of functional foods: A conjoint study of Danish, Finnish, and American consumers' perception of functional foods. Appetite 2003. 40(1): 9-14. DOI: https://doi.org/10.1016/S0195-6663(02)00171-X

9. Grunert K, Lähteenmäki, Boztug $Y$, Martinsdóttir E, Ueland $\varnothing$, Åström A, Lampila P: Perception of Health Claims Among Nordic Consumers. Journal of Consumer Policy 2009, 32: 269-287. DOI: https://doi.org/10.1007/s10603-009-9110-0

10. Krutulyte R, Grunert K, Scholderer J, Lähteenmäki L, Hagemann KS, Elgaard P, Nielsen B, Graverholt JP: Perceived fit of different combinations of carriers and functional ingredients and its effect on purchase intention. Food Quality and Preference 2011, 22(1): 1116. DOI:

https://doi.org/10.1016/j.foodqual.2010.06.001

11. Poulsen J: Danish consumers' attitudes towards functional foods. EconPapers 2001.

12. Temesi Á, Bascó Á, Grunert K, Lakner Z: Perceived Correspondence of Health Effects as a New Determinant Influencing Purchase Intention for Functional Food. Nutrients 2019, 11(4): 740. DOI: https://doi.org/10.3390/nu11040740

13. Huang L, Bai L, Gong S: The effects of carrier, benefit, and perceived trust in information channel on functional food purchase intention among Chinese consumers. Food Quality and Preference 2020, 81: 103854 DOI: https://doi.org/10.1016/j.foodqual.2019.103854

14. Plasek B, Temesi Á: The credibility of the effects of functional food products and consumers' willingness to purchase/willingness to pay- review. Appetite 2019, 143: 104398.

https://doi.org/10.1016/i.appet.2019.104398

15. Moons I, Barbarossa C, Pelsmacker PD: The Determinants of the Adoption Intention of Eco-friendly Functional Food in Different Market Segments. Ecological Economics 2018, 151: 151-161. DOI: https://doi.org/10.1016/j.ecolecon.2018.05.012

16. Hilton J: Chapter 1- Growth patterns and emerging opportunities in nutraceutical and functional food categories: market overview. Developing New Functional Food and Nutraceutical Products 2017, 1-28. DOI: $\quad$ https://doi.org/10.1016/B978-0-12-8027806.00001-8

17. Chen S, Martirosyan D: FOSHU-approved Fibersol ${ }^{\circledR}-2$ product review. Bioactive Compounds in Health and Disease 2021, 4(5): 79-89. DOI: https://www.doi.org/10.31989/bchd.v4i5.797

18. Saguy IS, Moskowitz HR: Integrating the consumer into new product development. Food technology 1999, 53(8): 68-73. 